\title{
Comparison of Radiographic and Surface Topography Measurements in Adolescents with Idiopathic Scoliosis
}

\author{
Jason M. Frerich ${ }^{\S, 1, *}$, Kristen Hertzler ${ }^{\S, 1}$, Patrick Knott ${ }^{1}$ and Steven Mardjetko ${ }^{2}$ \\ ${ }^{I}$ Rosalind Franklin University of Medicine and Science - Chicago Medical School, 3333 Green Bay Road, North \\ Chicago, IL 60064, USA \\ ${ }^{2}$ Illinois Bone and Joint Institute, 9000 Waukegan Road, Morton Grove, IL 60053, USA
}

\begin{abstract}
Purpose: In patients with adolescent idiopathic scoliosis (AIS), radiographic surveillance is the gold standard of assessing spinal deformity, but has negative long-term effects. The Formetric 4D surface topography system was compared to standard radiography as a safer option for evaluating patients with AIS.

Methods: Fourteen volunteers with typical AIS patient stature had 30 repeated Formetric 4D measurements taken, and reproducibility was assessed. Sixty-four patients with AIS were then enrolled during routine clinic visits. Evaluation included standard radiographs and surface topography measurements. A comparison analysis was performed.

Results: When assessing same-day repeated scans, a standard deviation of +/- 3.4 degrees for scoliosis curve measurements was determined, and the Reliability Coefficient (Cronbach) was very high (0.996). Cobb angles measured with the Formetric 4D differed from radiographic measurements by an average of 9.42 (lumbar) and 6.98 (thoracic) degrees, while the correlation between the two measurements was strong (95\% confidence interval [CI]), 0.758 (lumbar) and 0.872 (thoracic) respectively.

Conclusions: The Formetric 4D is comparable to radiography in terms of its test-retest reproducibility. Although this device does not predict curve magnitude exactly, the predictions correlate strongly with the Cobb angles determined from radiographs. It can be reliably used in the surveillance of patients with AIS.
\end{abstract}

Keywords: Scoliosis, radiography, surface topography, surveillance, comparison.

\section{INTRODUCTION}

Adolescent Idiopathic Scoliosis (AIS) is a structural spinal deformity in the coronal plane that affects $1-3 \%$ of children in the United States [1]. When the deformity is minor, less than $20^{\circ}$, treatment for this condition typically includes observation and surveillance to look for indications of curve progression [2]. Bracing and surgery are the treatments indicated for larger curves, greater than $20^{\circ}$ and $40^{\circ}$ respectively [2], though management in these patients also includes surveillance for evidence of a change in the deformity.

Scoliosis is usually found on a standard screening. The gold standard for diagnosis and subsequent curve surveillance remains standing full-column radiographs of the spine [3]. Radiographic images allow healthcare professionals to assess the dimensions of the deformity in both the coronal and sagittal planes, while quantifying the spinal curvatures by deriving Cobb angle measurements. The disadvantage of radiographs, particularly in young patients, however, is that repeat exposure to ionizing radiation causes a significant increase in the risk of malignancies later in life

*Address correspondence to this author at the Chicago Medical School, Rosalind Franklin University of Medicine and Science, 3333 Green Bay Road, North Chicago, IL 60064, USA; Tel: (515) 707-3553; Fax: (847) 5788690; E-mail: jason.frerich@my.rosalindfranklin.edu

${ }^{\S}$ Demarcates co-authorship.
$[4,5]$. The relative risk of breast cancer, for example, is nearly 4 times greater in these patients [6]. Nash et al. reported that in the late 1970's the average teenage girl with scoliosis received 22 radiographs over three years of surveillance for AIS [7]. The radiation dose for standard $\mathrm{x}$ rays has improved significantly over the years [8], but nonradiographic ways to image the spine and predict spinal deformity are still very important. While no patient can avoid x-rays completely, there should be an effort to reduce radiation exposure whenever possible.

Surface topography has been used for this purpose for many years, beginning with the use of the Scoliometer to measure trunk rotation in the late 1980's [9]. Many systems using surface topography have since been developed [10-25], but there has not yet been a system that has gained widespread approval. The published research on topography has shown inconsistent reliability as a method of measuring spinal deformity [18]. The goal of this study was to test a new system of surface topography and determine whether it was reliable and reproducible and, ultimately, evaluate whether it could produce Cobb angle measurements that could be dependably correlated with those obtained via traditional radiography.

The Formetric 4D system by Diers Medical Systems (225 Washington Street, Suite 2200, Chicago, IL 60606, www.diers.de) was developed in Germany and has been used extensively throughout several European countries for the last 5 years. As with other surface topography systems, it 
projects stripes of white light (raster lines) on the back of a standing patient and captures a digital photo of the image to assess pinpoint surface asymmetry and identify bony landmarks. In obese patients, external markers can be placed over landmarks felt beneath the skin by the clinician. The machine then compares the observed surface topography to a database of thousands of radiographic and topographic measurements of patients with scoliosis, utilizing a complex algorithm to quickly re-create a three dimensional representation of the patient's spine without exposing them to harmful radiation (Fig. 1). Recent literature has shown that the accuracy of these mathematical models has been improving over the years with the use of more powerful computers and more sophisticated formulas $[25,26]$. sway cycles. Each participant was female, between the ages of 16 and 25, with BMIs ranging from 17-25. Nine participants had no scoliosis or insignificant curves (curvature $<10^{\circ}$ ), and five had curves ranging in magnitude from $15^{\circ}$ to $40^{\circ}$ on recently taken radiographs.

Measurement protocol described by the manufacturer was followed for each measurement obtained, and participants were asked to stand in their normal, comfortable posture. Participants were asked to step away from the machine between measurements. The examiner did not position the participant, and there were no external markers placed. Thirty consecutive measurements were done on each patient, and there were no data sets excluded. Anatomical bony landmarks were detected automatically by the
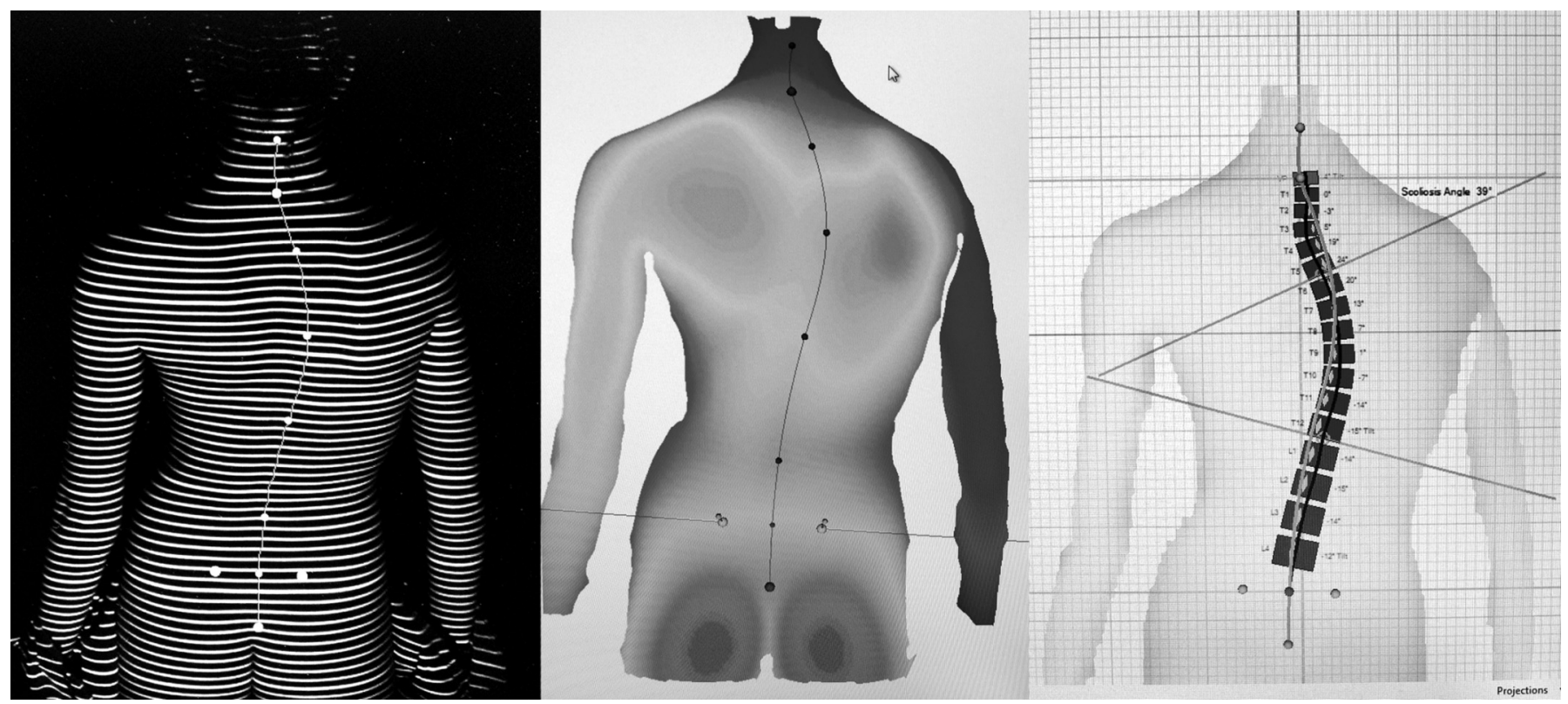

Fig. (1). Topographic analysis and software output from the Formetric 4D device.

Furthermore, the Formetric 4D device measures patients over a 6-second interval, taking 2 pictures per second. The 12 images acquired are evaluated and averaged by the machine's software, correcting for any subject movement during the data acquisition period. The model generated by the algorithm can then be used to calculate the Cobb angle of a scoliosis curve, and the patient can undergo repeated scans over time as a method of surveillance for curve progression. Ideally, when the Formetric 4D device predicts that curve progression has occurred, a radiograph can subsequently be taken to then confirm the change.

\section{METHODS}

Two separate studies were conducted. The reliability and reproducibility of the Formetric 4D measurements were initially evaluated, followed by a comparison analysis between the Formetric 4D measurements and standard radiographic measurements. An IRB was approved for both studies. In assessing reliability and reproducibility, 14 volunteer participants were enrolled and assigned to have 30 Formetric 4D scans completed over the course of a 60minute time period. A six-second acquisition time was selected in order to sufficiently account for average postural
Formetric 4D system, and the examiner did not manually change any of the landmark locations that were selected by the machine.

For each set of 30 measurements, there were ten parameters that were evaluated: Trunk Length, Trunk Imbalance, Pelvic Tilt, Thoracic Kyphosis, Lumbar Lordosis, Maximal Right Trunk Rotation, Maximal Left Trunk Rotation, Maximal Apical Deviation to the Right, Maximal Apical Deviation to the Left, and Scoliosis Angle. Standard deviation was calculated for each of these 10 parameters, and an ANOVA for Repeated Measures was determined for this data set.

Once reproducibility was evaluated, it was necessary to determine whether the Formetric 4D measurements could be reliably compared to those obtained from x-ray. Sixty-four patients, 9 male and 55 female, between the ages of 9-17, who were being regularly evaluated for AIS, were enrolled during routine clinic visits for comparison analysis. Each patient had a diagnosis of AIS and scoliosis curves (measured as Cobb angles on radiographs) ranging between 10 and 50 degrees. Patient BMIs varied between 16 and 20 . 
Each patient first received a standard posteroanterior radiograph as part of their clinic visit and then underwent back-only surface topographic analysis using the Formetric 4D system. As before, the procedure described by the manufacturer was followed while obtaining the measurements, and the patients were only asked to stand in their usual, comfortable position. All radiographs taken were evaluated by an experienced orthopedist and Cobb angles were determined for both coronal and sagittal curves using standard techniques. Similar measurements were generated by the Formetric 4D device, and a comparison analysis was made. Two of the patients were measured twice, on separate office visits, for a total of 66 sets of data. There were a total of 50 lumbar scoliosis curves and 52 thoracic scoliosis curves measured, as well as 61 measurements of thoracic kyphosis and lumbar lordosis within the sagittal plane.

For each set of patient data, there were four parameters compared: thoracic scoliosis curvature, lumbar scoliosis curvature, thoracic kyphosis, and lumbar lordosis. A Pearson correlation coefficient was calculated for each of the four parameters to compare the two measurement systems. The average difference, standard deviation, and range of difference were also determined for each parameter.

\section{RESULTS}

The average standard deviations found for the 10 parameters analyzed in assessing reliability and reproducibility are listed in Table 1. When evaluating same-day repeat scans, a standard deviation of $+/-3.4$ degrees for scoliosis curve measurements was determined. An ANOVA for Repeated Measures (StatPlus version 5.8, AnalySoft, Inc.) showed that the Reliability
Coefficient (Cronbach's Alpha) for the scoliosis measurement was 0.996, indicating that for each patient measured the scoliosis angle calculated by the Formetric 4D was very reliable. Additionally, the parameters assessed when comparing the Formetric 4D measurements to those obtained from x-ray (average difference, standard deviation, range of difference, and correlation) for the 66 sets of radiographic and topographic data are summarized in Table 2.

The correlations found when comparing the Formetric 4D measurements to radiographic measurements were strong $(>0.700)$ and statistically significant $(\mathrm{p}<0.0001$ at the level of significance Alpha $=0.05$ ) for all curve types measured (Fig. 2-5). The Formetric 4D reliably predicted spinal curvature at all levels in both coronal and sagittal planes. However, in comparison to radiograph, the Formetric 4D device underestimated the magnitude of curves measured by an average of 8.12 degrees in all parameters except thoracic kyphosis, in which it consistently overestimated the magnitude by an average of 7.26 degrees.

\section{DISCUSSION}

Since the invention of the Scoliometer, there has been a push towards finding a reliable and effective means of utilizing surface topography to follow spinal curve progression in patients with AIS. Several devices which utilize surface topography have been developed, however, none of them have gained widespread approval [10-25]. Clinicians may be hesitant to rely on an alternative to radiography for obtaining data that determines whether a patient receives drastic therapy, such as surgery. This is likely secondary to the lack of consistent reproducibility and

Table 1. Parameters Measured in Assessing Formetric 4D Reproducibility

\begin{tabular}{|l|c|c|}
\hline \multicolumn{1}{|c|}{ Parameter } & Average Standard Deviation & Range \\
\hline \hline Trunk Length & $5.4 \mathrm{~mm}$ & $2.5-11.1$ \\
\hline Trunk Imbalance & $5.1 \mathrm{~mm}$ & $2.8-7.2$ \\
\hline Pelvic Tilt & 2.2 degrees & $1.2-4.8$ \\
\hline Thoracic Kyphosis & 2.7 degrees & $1.0-4.3$ \\
\hline Lumbar Lordosis & 2.1 degrees & $1.2-4.2$ \\
\hline Maximal Right Rotation & 2.4 degrees & $1.0-3.8$ \\
\hline Maximal Left Rotation & 1.6 degrees & $0.7-3.1$ \\
\hline Max Right Lateral Deviation & $2.3 \mathrm{~mm}$ & $1.2-4.3$ \\
\hline Max Left Lateral Deviation & $2.1 \mathrm{~mm}$ & $1.4-3.9$ \\
\hline Scoliosis Angle of Major Curve & $3.4 \mathrm{degrees}$ & $1.2-6.2$ \\
\hline
\end{tabular}

Table 2. Parameters Analyzed when Comparing Formetric 4D Measurements to those Obtained from X-Ray

\begin{tabular}{|c|c|c|c|c|}
\hline $\begin{array}{c}\text { Parameter } \\
\text { Measured }\end{array}$ & $\begin{array}{c}\text { Number of } \\
\text { Curves Measured }\end{array}$ & $\begin{array}{c}\text { Average Difference Between Radiograph } \\
\text { and Topography Measurement }\end{array}$ & Range of Differences & $\begin{array}{c}\text { Correlation (r) Between } \\
\text { Radiograph \& Topography }\end{array}$ \\
\hline \hline Lumbar Curve & 50 & 9.40 degrees & $0-22$ & 0.758 \\
\hline Thoracic Curve & 52 & 7.00 degrees & $0-19$ & 0.872 \\
\hline Thoracic Kyphosis & 62 & 10.6 degrees & $1-24$ & 0.799 \\
\hline Lumbar Lordosis & 62 & 8.00 degrees & $0-21$ & 0.813 \\
\hline
\end{tabular}


comparability previously found in studies assessing spinal imaging devices that utilize surface topography.

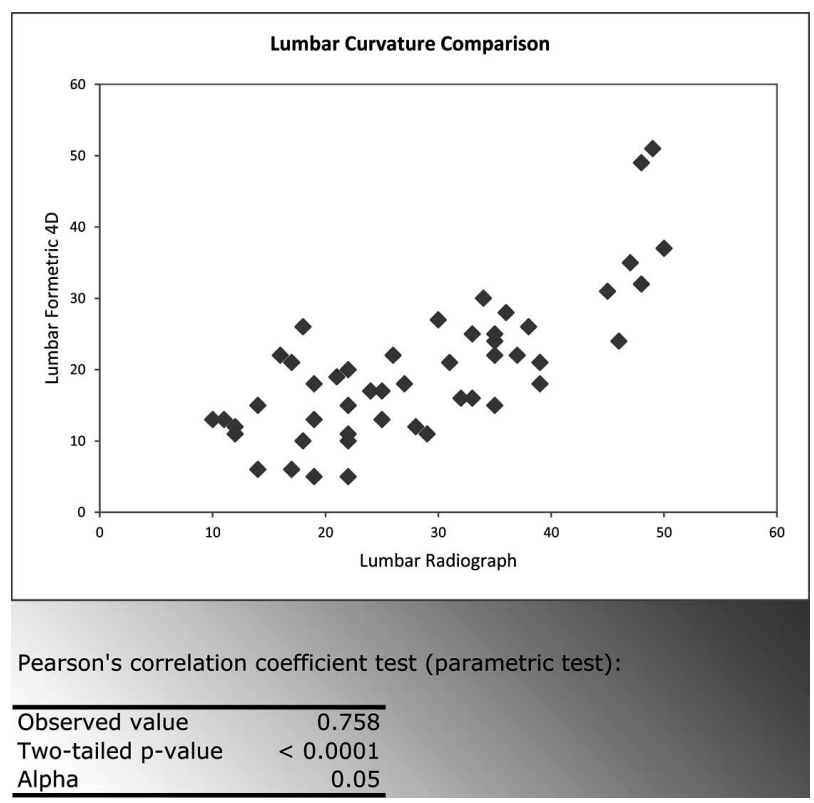

Fig. (2). Lumbar Curvature Formetric 4D vs Radiograph.

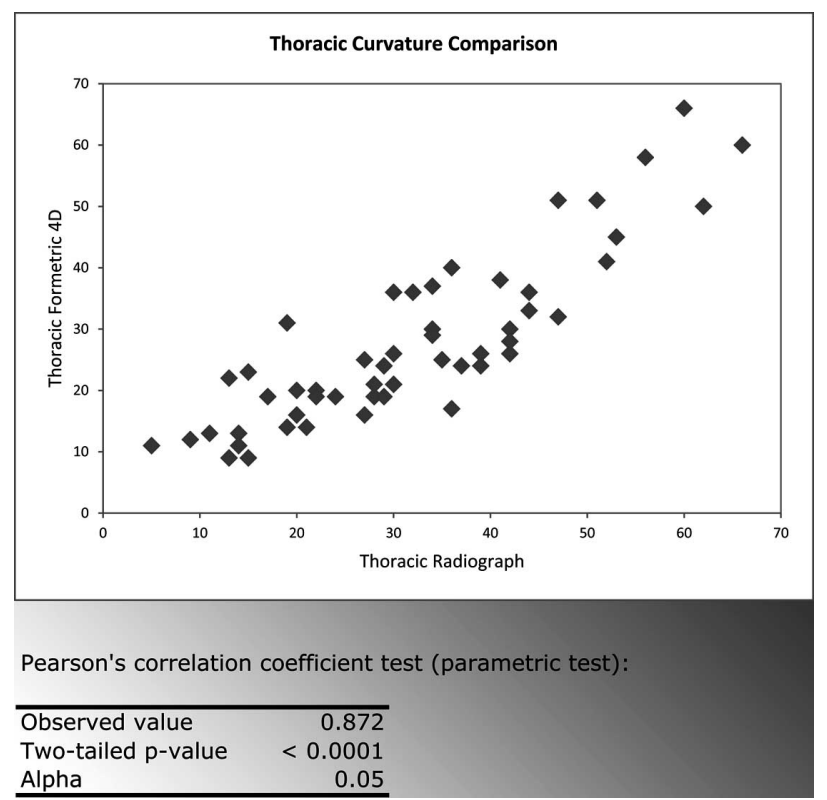

Fig. (3). Thoracic Curvature Formetric 4D vs Radiograph.

Recent studies have demonstrated an intra-observer variance of 5.14 degrees and an inter-observer variance of 6.54 degrees when utilizing standard radiographs to determine Cobb angle measurements [26]. When assessing same-day repeat scans, this study found that the average standard deviation for the Formetric 4D scoliosis curve measurements (+/- 3.4 degrees) was consistent with the variance found when either two clinicians interpret the same radiograph or one clinician interprets the same radiograph twice. This makes the device a reliable tool, comparable to radiographs, in terms of its test-retest reproducibility. One limitation with this segment of the study, however, was the small number of patients assessed. Each patient was measured 30 times, and 420 data sets were used in an attempt to mitigate this problem.

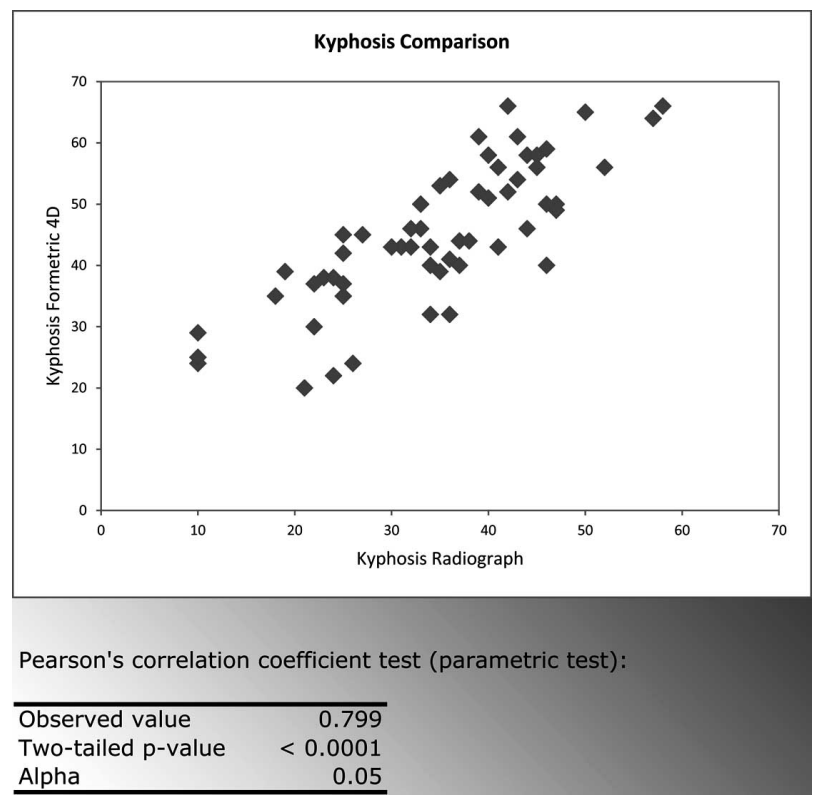

Fig. (4). Kyphosis Formetric 4D vs Radiograph.

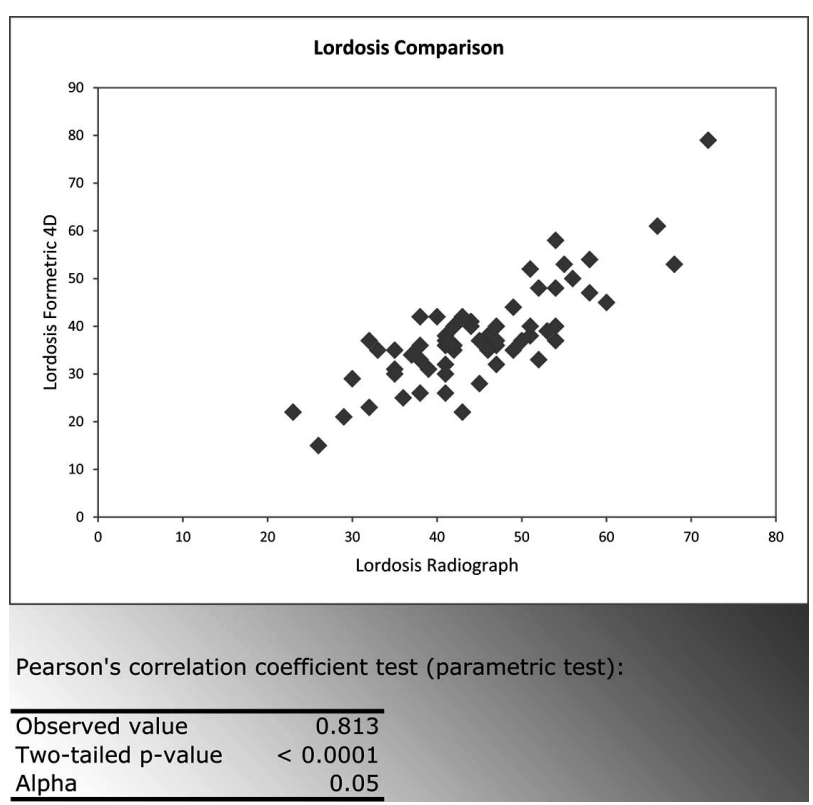

Fig. (5). Lordosis Formetric 4D vs Radiograph.

Most significantly, it was determined that Cobb angle measurements obtained from AIS patients using the Formetric 4D system strongly correlated with those obtained by standard radiographs. Although this device does not predict curve magnitude exactly, it is not necessary for the surface topography and radiographic measurements to be the same, as the purpose of topography is primarily to identify a change. It is this change in topography that will alert the clinician to the possibility of progression in the actual scoliosis curve. The authors conclude that the Formetric 4D can be reliably used in the surveillance of patients with AIS. 
However, further studies are certainly needed to confirm the long-term effectiveness of using the Formetric 4D in clinical decision-making. At this time, a multi-center prospective study is underway to evaluate the Formetric 4D's predicted ability, in comparison to radiograph, to accurately monitor curve progression over a two-year interval in patient's being routinely followed with AIS.

Surface topography will not completely replace radiographic analysis in monitoring patients with AIS, as it cannot evaluate the actual bone morphology the way a radiograph can. However, it has obvious advantages to repeat radiographs in the adolescent population, importantly the reduction in exposure to ionizing radiation. If it can deliver reliable and comparable results, then it should replace radiographs during clinic visits when curve surveillance is necessary but exposure to radiation can be avoided. Identified topographic changes can then be followed up with radiographic imaging to confirm curve progression and determine therapeutic intervention.

\section{ACKNOWLEDGEMENTS}

The authors have no conflicts of interest to disclose. No funds were received for the performance of this research, although the Formetric 4D machine was loaned to the Illinois Bone and Joint Institute for this evaluation.

\section{CONFLICT OF INTEREST}

The author(s) confirm that this article content has no conflicts of interest.

\section{REFERENCES}

[1] Soucacaos PN, Zacharis K, Soultanis K, Gelalis J, Xenakis T, Beris AE. Risk factors for idiopathic scoliosis: review of a 6-year prospective study. Orthopedics 2000; 23(8): 833-8.

[2] Yawn BP, Yawn RA. The estimated cost of school scoliosis screening. Spine (Phila Pa 1976) 2000; 25(18): 2387-91.

[3] Raso VJ, Lou E, Hill DL, Mahood JK, Moreau MJ, Durdle NG. Trunk distortion in adolescent idiopathic scoliosis. J Pediatr Orthop 1998; 18(2): 222-6.

[4] Boice JD, Jr. Carcinogenesis--a synopsis of human experience with external exposure in medicine. Health Phys 1988; 55(4): 621-30.

[5] Morin DM, Lonstein JE, Stovall M, Hacker DG, Luckyanov N, Land CE. Breast cancer mortality after diagnostic radiography: findings from the U.S. Scoliosis Cohort Study. Spine (Phila Pa 1976) 2000; 25(31): 2052-63.

[6] Ronckers CM, Land CE, Miller JS, Stovall M, Lonstein JE, Doody MM. Cancer mortality among women frequently exposed to radiographic examinations for spinal disorders. Radiat Res 2010; 174(1): 83-90.

[7] Nash CL, Jr., Gregg EC, Brown RH, Pillai K. Risks of exposure to $\mathrm{X}$-rays in patients undergoing long-term treatment for scoliosis. $\mathrm{J}$ Bone Joint Surg Am 1979; 61(3): 371-4.
[8] Huda W, Nickoloff EL, Boone JM. Overview of patient dosimetry in diagnostic radiology in the USA for the past 50 years. Med Phys 2008; 35(12): 5713-28.

[9] Upadhyay SS, Burwell RG, Webb JK. Hump changes on forward flexion of the lumbar spine in patients with idiopathic scoliosis. A study using ISIS and the Scoliometer in two standard positions. Spine (Phila Pa 1976) 1988; 13(2): 146-51.

[10] Pearson JD, Dangerfield PH, Atkinson JT, et al. Measurement of body surface topography using an automated imaging system. Acta Orthop Belg 1992; 58(Suppl. 1): 73-9.

[11] Batouche M, Benlamri R, Kholladi MK. A computer vision system for diagnosing scoliosis using moiré images. Comput Biol Med 1996; 26(4): 33-53.

[12] Oxborrow NJ. Assessing the child with scoliosis: the role of surface topography. Arch Dis Child 2000; 83(5): 453-5.

[13] Macdonald AM, Griffiths CJ, MacArdle FJ, Gibson MJ. The effect of posture on Quantec measurements. Stud Health Technol Inform 2002; 91: 190-3.

[14] Hill DL, Berg DC, Raso VJ, et al. Evaluation of a laser scanner for surface topography. Stud Health Technol Inform 2002; 88: 90-4.

[15] Treuillet S, Lucas Y, Crepin G, Peuchot B, Pichaud JC. SYDESCO: a laser-video scanner for 3D scoliosis evaluations. Stud Health Technol Inform 2002; 88: 70-3.

[16] Liu XC, Thometz JG, Lyon RM, McGrady L. Effects of trunk position on back surface-contour measured by raster stereophotography. Am J Orthop 2002; 31(7): 402-6.

[17] Pazos V, Cheriet F, Song L, Labelle H, Dansereau J. Accuracy assessment of human trunk surface 3D reconstructions from an optical digitizing system. Med Biol Eng Comput 2005; 43(1): 11-5.

[18] Knott P, Mardjetko S, Nance D, Dunn M. Electromagnetic topographical technique of curve evaluation for adolescent idiopathic scoliosis. Spine (Phila Pa 1976) 2006; 31(24): E911-5.

[19] Goldberg CJ, Grove D, Moore DP, Fogarty EE, Dowling FE. Surface Topography and vectors: a new measure for the three dimensional quantification of scoliotic deformity. Stud Health Technol Inform 2006; 123: 449-55.

[20] Mitchell H, Pritchard S, Hill D. Surface alignment to unmask scoliotic deformity in surface topography. Stud Health Technol Inform 2006; 123: 109-16.

[21] Zubovic A, Davies N, Berryman F, et al. New Method of Scoliosis Deformity Assessment: ISIS2 System. Stud Health Technol Inform 2008; 140: 157-60.

[22] Shannon TM. Development of an apparatus to evaluate Adolescent Idiopathic Scoliosis by dynamic surface topography. Stud Health Technol Inform 2008; 140: 121-7.

[23] Berryman F, Pynsent P, Fairbank J. Measuring the rib hump in scoliosis with ISIS2. Stud Health Technol Inform 2008; 140: 65-7.

[24] Fortin C, Feldman DE, Cherlet F, Labelle H. Validity of a quantitative clinical measurement tool of trunk posture in idiopathic scoliosis. Spine (Phila Pa 1976) 2010; 35(19): E988-94.

[25] Parent EC, Damaraju S, Hill DL, Lou E, Smetaniuk D. Identifying the best surface topography parameters for detecting idiopathic scoliosis curve progression. Stud Health Technol Inform 2010; 158: 78-82.

[26] He JW, Yan ZH, Liu J, et al. Accuracy and repeatability of a new method for measuring scoliosis curvature. Spine (Phila Pa 1976) 2009; 34(9): E323-9. 